\title{
Exploring the Role of Solvent on Carbohydrate-Aryl Interactions by Diffusion NMR-Based Studies
}

Linda Jütten, ${ }^{\dagger}$ Karla Ramírez-Gualito, ${ }^{\ddagger}$ Andreas Weilhard, ${ }^{\dagger}$ Benjamin Albrecht, ${ }^{\dagger}$ Gabriel Cuevas, ${ }^{\S}$

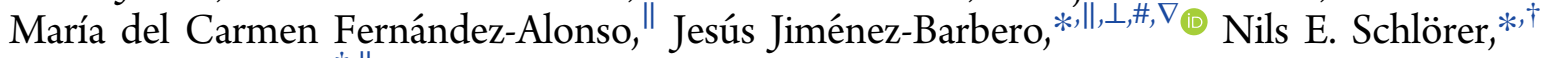
and Dolores Diaz ${ }^{*}, \dagger, \|$

${ }^{\dagger}$ Department für Chemie, NMR-Abteilung, Universität zu Köln, Greinstr. 4, 50939 Köln, Germany

${ }^{\ddagger}$ Centro de Nanociencias y Micro y Nanotecnología, Instituto Politécnico Nacional, Avenida Luis Enrique Erro S/N, Unidad Profesional Adolfo López Mateos, Zacatenco, C.P. 07738 Ciudad de México, México

${ }^{\S}$ Instituto de Química, Universidad Nacional Autónoma de México, Circuito Exterior, Ciudad Universitaria, Delegación Coyoacán, C.P. 04510 Ciudad de México, México

"Centro de Investigaciones Biológicas (CIB-CSIC), Ramiro de Maeztu 9, 28040 Madrid, Spain

${ }^{\perp}$ CIC bioGUNE, Science and Technology Park bld 801 A, 48160 Derio, Spain

"Basque Foundation for Science, Ikerbasque, Maria Diaz de Haro 3, 48013 Bilbao, Spain

${ }^{\nabla}$ Department of Organic Chemistry II, Faculty of Science \& Technology, University of the Basque Country, 48940 Leioa, Spain

Supporting Information

ABSTRACT: Carbohydrate-protein interactions play an important role in many molecular recognition processes. An exquisite combination of multiple factors favors the interaction of the receptor with one specific type of sugar, whereas others are excluded. Stacking $\mathrm{CH}$-aromatic interactions within the binding site provide a relevant contribution to the stabilization of the resulting sugar-protein complex. Being experimentally difficult to detect and analyze, the key $\mathrm{CH}-\pi$ interaction features have been very often dissected using a variety of techniques and simple model systems. In the present work, diffusion NMR spectroscopy has been employed to separate the components of sugar mixtures in different solvents on the basis of their differential ability to interact through $\mathrm{CH}-\pi$ interactions with one particular aromatic
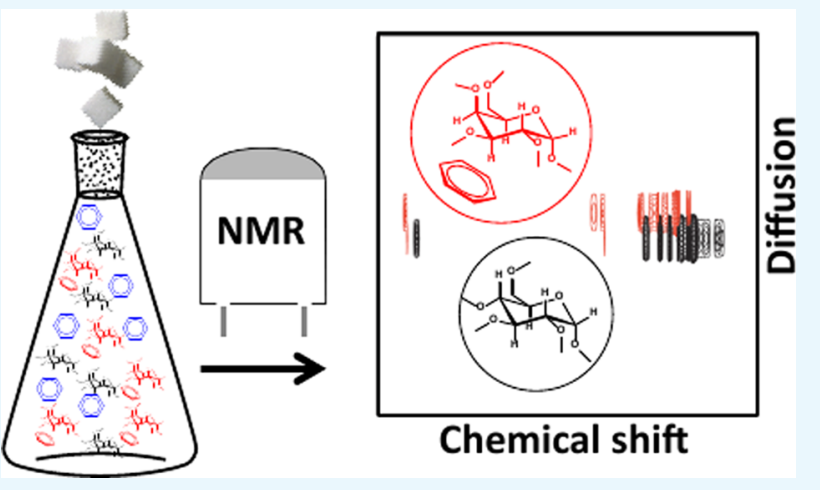
cosolute in solution. The experimental data show that the properties of the solvent did also influence the diffusion behavior of the sugars present in the mixture, inhibiting or improving their separation. Overall, the results showed that, for the considered monosaccharide derivatives, their diffusion coefficient values and, consequently, their apparent molecular sizes and/or shapes depend on the balance between solute/cosolute as well as solute/solvent interactions. Thus, in certain media and in the presence of the aromatic cosolute, the studied saccharides that are more suited to display $\mathrm{CH}-\pi$ interactions exhibited a lower diffusion coefficient than the noncomplexing sugars in the mixture. However, when dissolved in another medium, the interaction with the solvent strongly competes with that of the aromatic cosolute.

\section{INTRODUCTION}

Many biological events are initiated by molecular recognition processes that are triggered by the initial interaction between an exogenous/endogenous sugar-type ligand and membrane cell receptors. ${ }^{1}$ Although the formation of glycan/protein complexes is driven by many factors (e.g., hydrogen bond, van der Waals forces, etc.), the role of $\mathrm{CH}-\pi$ interactions in such a process is currently receiving special attention because it may subtly tune the properties of the ligand-protein complexes. Thus, substantial efforts are being made to analyze the features that promote such interactions between carbohydrate entities and the aromatic side chains from the residues within the recognition site. ${ }^{2}$

Detailed X-ray crystallographic structures of sugar/protein complexes have clearly shown the stabilizing contribution of $\mathrm{CH}-\pi$ interactions in these systems, ${ }^{3}$ whereas their detection in solution by NMR spectroscopy has also been achieved. ${ }^{4}$ Moreover, NMR methods have been employed to directly detect intramolecular methyl $-\pi$ interactions within a protein. ${ }^{5}$

Received: October 24, 2017

Accepted: December 28, 2017

Published: January 17, 2018 


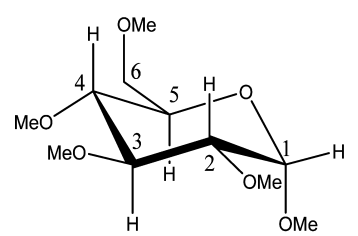

$\alpha-p M e G l c(1)$

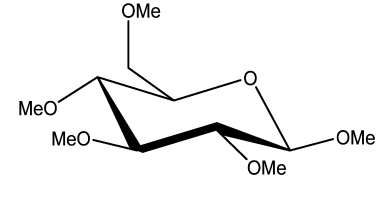

$\beta-p M e G l c(2)$

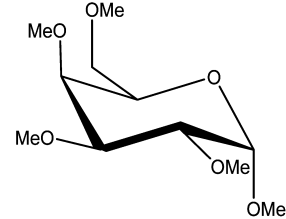

$\alpha-p M e G a l(3)$

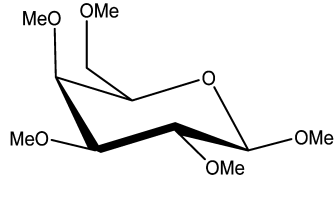

$\beta-p M e G a l(4)$

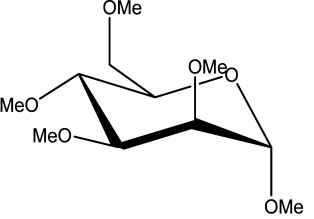

$\alpha-p M e M a n(5)$

Figure 1. Compound structures, name abbreviations, and atom numbering used in the present study: $\alpha$-pMeGlc $(1)=2,3,4,6$-tetra- $O$-methyl- $\alpha$-Dmethylglucopyranoside, $\beta$-pMeGlc(2) = 2,3,4,6-tetra- $O$-methyl- $\beta$-D-methylglucopyranoside, $\alpha$-pMeGal $(3)=2,3,4,6$-tetra- $O$-methyl- $\alpha$-D-methylgalactopyranoside, $\beta$-pMeGal(4) $=$ 2,3,4,6-tetra- $O$-methyl- $\beta$-D-methylgalactopyranoside, and $\alpha$-pMeMan $(5)=2,3,4,6$-tetra- $O$-methyl- $\alpha$-Dmethylmannopyranoside.

NMR spectroscopy has also been essential for the detection of $\mathrm{CH}-\pi$ interactions in model systems using simple solutions consisting of one particular monosaccharide and one aromatic moiety in aqueous medium. Hence, the shielding of specific sugar NMR signals in the presence of the aromatic compounds, ${ }^{6}$ as well as the detection of intermolecular nuclear Overhauser effects between these species in solution, ${ }^{7}$ have been correlated with the formation of sugar-aromatic complexes. The role of solvent on the formation of noncovalent $\mathrm{CH}-$ aromatic interactions has been investigated, focusing on the analysis of certain solvent parameters that were correlated with the modulation of the observed solute-solute $\mathrm{CH}-\pi$ interactions, ${ }^{8}$ suggesting that the interplay between solute/ solvent may have a modulating effect on these interactions ${ }^{9}$

Because optimal sugar-aromatic interactions involve a specific spatial arrangement of $\mathrm{CH}$ bonds within the sugar skeleton, ${ }^{6,7}$ some sugar moieties are more prone to establish stabilizing $\mathrm{CH}-\pi$ interactions (e.g., $\alpha / \beta$-galactose) than others (e.g., $\alpha$-mannose). Therefore, we hypothesized that different monosaccharides could be discriminated taking advantage of this recognition phenomenon. Herein, we detail our investigations to detect sugar-aromatic complexes within a sugar mixture by using diffusion-ordered spectroscopy (DOSY) NMR.

During the last decades, DOSY NMR has been proposed as a versatile tool to perform not only the virtual separation of complex mixtures but also to explore many other interesting aspects related to solute/solute and solute/solvent interactions associated with size and shape changes. ${ }^{10}$ In this respect, remarkable examples of applications of DOSY have been reported, including folding/unfolding of proteins, ${ }^{11}$ identification of secondary structure elements of nucleic acids, ${ }^{12}$ detection and identification of reaction intermediates, ${ }^{13}$ aggregation, ${ }^{14}$ hydrogen bonding, ${ }^{15}$ exchange, ${ }^{16}$ metal sugar complexation, ${ }^{17}$ host-guest complexation, ${ }^{18}$ detection of impurities, ${ }^{19}$ assessment of fraud in pharmaceutical formulations, ${ }^{20}$ estimation of the molecular weight of small molecules ${ }^{21}$ and large biomolecules, ${ }^{22}$ among many others.

A number of strategies have been proposed to enhance the performance of DOSY NMR experiments, mostly based on subtle modifications of the medium to modify the diffusivity of the molecules. Hence, the difficult separation of a mixture of isomers with identical molecular weight has been accomplished by switching solvents. ${ }^{23}$ The so-called "matrix-assisted" DOSY method did allow the discrimination between epimers by using a (chiral) co-adjuvant that interacts specifically with only one component of the mixture and selectively modifies its translational diffusion properties. ${ }^{24}$ The use of chromatographic adsorbents has also been demonstrated to efficiently facilitate the separation of complex mixtures using solid-state NMR ("NMR chromatography"). ${ }^{25}$

Herein, we provide new evidences for the intricate interplay of the solvent properties that influence the strength of $\mathrm{CH}-\pi$ interactions between sugars and aromatic compounds. The use of DOSY NMR has permitted investigating the influence of the solvent on both the separation of the different sugars (diastereomers) in mixtures as well as for the discrimination of those sugars that display intrinsic propensities to form noncovalent complexes with aromatic molecules.

\section{RESULTS AND DISCUSSION}

A series of permethylated monosaccharides known to be soluble in a variety of solvents ranging from nonpolar lipophilic cyclohexane to polar protic water were synthesized ${ }^{26}$ (Figure 1, compounds 1-5). They were considered to be suitable model systems to investigate carbohydrate/aryl interactions, as well as those solvent properties that might modulate the separation of mixtures of sugar diastereomers, as has been described for regioisomers of simple molecules. ${ }^{23}$

The diffusion properties of the components of equimolar binary mixtures of sugars $\mathbf{1 - 5}$, as well as the role of the solvent properties (i.e., cyclohexane, chloroform, and water), were first investigated.

For a proper performance of DOSY NMR experiments, a scarce dispersion of the signals in the NMR spectrum of mixtures is clearly an obstacle but, in this case, the isolated signals in the studied mixtures were sufficient to unambiguously identify the diffusion coefficient values of each of their components. The one-dimensional (1D) ${ }^{1} \mathrm{H}$ NMR spectrum of a representative mixture in water is shown in Figure 2. As typically occurs with carbohydrates, most of the signals overlap within a narrow region of the spectrum, including in this case, the signals of the ring protons and also the singlets of the methyl substituents. Fortunately, for most of the analyzed mixtures, the anomeric signals and some ring proton signals from each sugar within the mixtures were clearly distinguishable.

Role of the Solvent. DOSY NMR has been already employed to separate carbohydrate mixtures that differ by molecular weight (oligo and polysaccharides), ${ }^{27}$ branching pattern, ${ }^{28}$ and/or shape. ${ }^{29}$ However, to our knowledge, until now there has not been any study on the potential of diffusion NMR to separate mixtures of sugar diastereomers and on the possible influence of solvent properties to achieve this aim through specific solute/solvent interactions.

In this context, we first explored whether the separation of the components of binary mixtures of sugars $1-5$ could be 


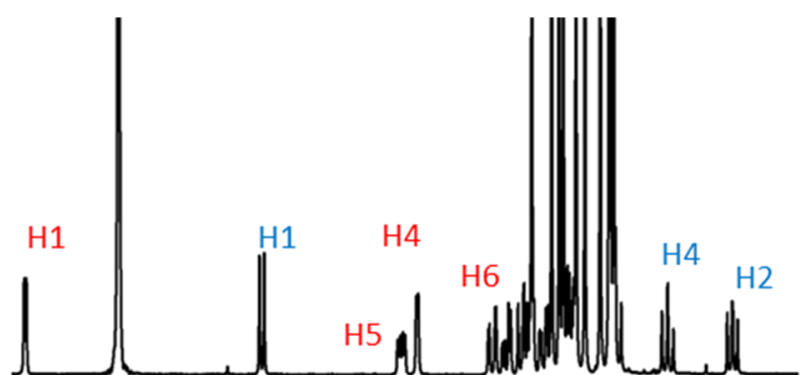

\begin{tabular}{llllllllllll}
\hline & & & & & & & & & & & \\
$\delta^{1} \mathrm{H}$ & 4.8 & 4.6 & 4.4 & 4.2 & 4.0 & 3.8 & 3.6 & 3.4 & 3.2 & $\mathrm{ppm}$
\end{tabular}

Figure 2. One-dimensional ${ }^{1} \mathrm{H}$ NMR spectrum of an equimolar mixture of $\beta$-pMeGlc (2)/ $\alpha$-pMeGal(3) in $\mathrm{D}_{2} \mathrm{O}$ at $298 \mathrm{~K}$ (ca. $25 \mathrm{mM}$, $600 \mathrm{MHz}$ ). Assignments of the signals of $\beta$-pMeGlc(2) and $\alpha$ $\mathrm{pMeGal}(3)$ are highlighted with blue (2) and red (3).

achieved exclusively by switching solvents with different properties.

In Figure 3, the DOSY spectra of the mixture $\alpha$-pMeGlc(1)/ $\alpha$-pMeGal(3) in different solvents are shown. It is noticeable that the diffusion coefficient values of the components of the mixture, as well as the mixture separation, depend significantly on the medium properties.

The mixture in aqueous solution could not be separated as the components showed the same self-diffusion coefficient $D_{\mathrm{D}_{2} \mathrm{O}}(\mathbf{1})=D_{\mathrm{D}_{2} \mathrm{O}}(3)=5.25 \times 10^{-10} \mathrm{~m}^{2} \mathrm{~s}^{-1}$ (Figure $3 \mathrm{c}$ ). In cyclohexane and chloroform, however, $\alpha$-pMeGlc $(1)$ and $\alpha$ pMeGal(3) exhibited slight, although evident, differences in their respective diffusion coefficient values (Figure 3a,b). Interestingly, although in cyclohexane, the apparent size of $\alpha$ $\operatorname{pMeGlc}(\mathbf{1})$ is smaller than that of $\alpha$-pMeGal$(3)\left(D_{\mathrm{C}_{6} \mathrm{D}_{12}}(\mathbf{1})=1\right.$ $\times 10^{-9} \mathrm{~m}^{2} \mathrm{~s}^{-1}$ and $\left.D_{\mathrm{C}_{6} \mathrm{D}_{12}}(3)=9.33 \times 10^{-10} \mathrm{~m}^{2} \mathrm{~s}^{-1}\right)$, in chloroform, the opposite occurred and $\alpha$-pMeGlc $(1)$ had a lower self-diffusion coefficient $\left(D_{\mathrm{CDCl}_{3}}(1)=4.78 \times 10^{-10} \mathrm{~m}^{2}\right.$ $\left.\mathrm{s}^{-1}\right)$ than that of $\alpha$-pMeGal$(3)\left(D_{\mathrm{CDCl}_{3}}(3)=5.13 \times 10^{-10} \mathrm{~m}^{2}\right.$ $\left.\mathrm{s}^{-1}\right)$.

Figure 4 shows the DOSY spectra of several binary mixtures of 1-5 in cyclohexane- $d_{12}$. Fittingly, most of the studied mixtures are separated and their components (diastereomers) showed slightly although clearly distinctive diffusion coefficient values in cyclohexane.
For instance, paying attention to the $\alpha$-pMeGlc $(1) / \beta$ pMeGal(4) mixture (Figure 4a) and according to the Stokes-Einstein equation, $\alpha$-pMeGlc(1) displays the apparent smallest hydrodynamic radius (largest diffusion coefficient, $\left.D_{\mathrm{C}_{6} \mathrm{D}_{12}}(1)=1 \times 10^{-9} \mathrm{~m}^{2} \mathrm{~s}^{-1}\right)$ in comparison to that of any of the galactose derivatives $\left(D_{\mathrm{C}_{6} \mathrm{D}_{12}}(3)=D_{\mathrm{C}_{6} \mathrm{D}_{12}}(4)=9.33 \times 10^{-10}\right.$ $\left.\mathrm{m}^{2} \mathrm{~s}^{-1}\right)$. The $\alpha / \beta$ configuration at the anomeric position of the galactose derivatives did not seem to have any influence (Figures $3 \mathrm{a}$ and $4 \mathrm{a}$ ). The difference between the glucose and galactose diastereomers resides at the orientation of the methoxy group (equatorial and axial, respectively) at $\mathrm{C} 4$, which in turn also affects the distribution of rotamers around the C5-C6 torsions. It has been established that this distribution is also modulated by the solvent properties. ${ }^{30}$ Thus, we initially reasoned that the axial orientation of $\mathrm{O} 4$ could be at the origin of the apparently larger size of the galactose derivatives when compared with the glucose analogues in cyclohexane.

The DOSY spectrum of the $\beta$-pMeGlc(2)/ $\alpha$-pMeMan(5) mixture (Figure $4 \mathrm{~b})$ showed the same trend $\left(D_{\mathrm{C}_{6} \mathrm{D}_{12}}(2)=1 \times\right.$ $10^{-9} \mathrm{~m}^{2} \mathrm{~s}^{-1}$ and $D_{\mathrm{C}_{6} \mathrm{D}_{12}}(5)=9.33 \times 10^{-10} \mathrm{~m}^{2} \mathrm{~s}^{-1}$, respectively), confirming that the configuration at the anomeric position is not critical but rather the configuration at position $\mathrm{C} 2(\alpha$ $\mathrm{pMeMan}(5)$ with axial O2) or $\mathrm{C} 4(\beta$-pMeGal(4) with axial $\mathrm{O} 4)$ is crucial for the separation. Because the distribution of the C5-C6 rotamers for mannose and glucose are analogous, ${ }^{31}$ we concluded that the observed separation and associated size differences in the pMeGlc/pMeGal and pMeGlc/pMeMan mixtures were mostly driven by the presence of axial substituents $(\mathrm{C} 2 / \mathrm{C} 4)$ in the $\mathrm{Gal}$ and Man moieties. The absence of separation in the mixtures $\alpha$-pMeGal(3)/ $\alpha$ pMeMan(5) (Figure 4c) supported this hypothesis (identical diffusion coefficient values $D_{\mathrm{C}_{6} \mathrm{D}_{12}}(3)=D_{\mathrm{C}_{6} \mathrm{D}_{12}}(\mathbf{5})=9.33 \times 10^{-10}$ $\left.\mathrm{m}^{2} \mathrm{~s}^{-1}\right)$. Thus, the simple presence of axial substituents at $\mathrm{C} 2$ or C4 strongly modulates the diffusion properties and the apparent sizes of these permethylated monosaccharides (15) in the nonpolar cyclohexane.

As the next step, the DOSY NMR spectra of selected mixtures of $\mathbf{1 - 5}$ in aqueous solution were also analyzed. The results obtained for the mixtures in water were initially confusing because none of them could be separated by DOSY NMR and all of them exhibited the same diffusion coefficient value $\left(D_{\mathrm{D}_{2} \mathrm{O}}(1-5)=5.25 \times 10^{-10} \mathrm{~m}^{2} \mathrm{~s}^{-1}\right)$. We thus concluded that in contrast to the interactions in the

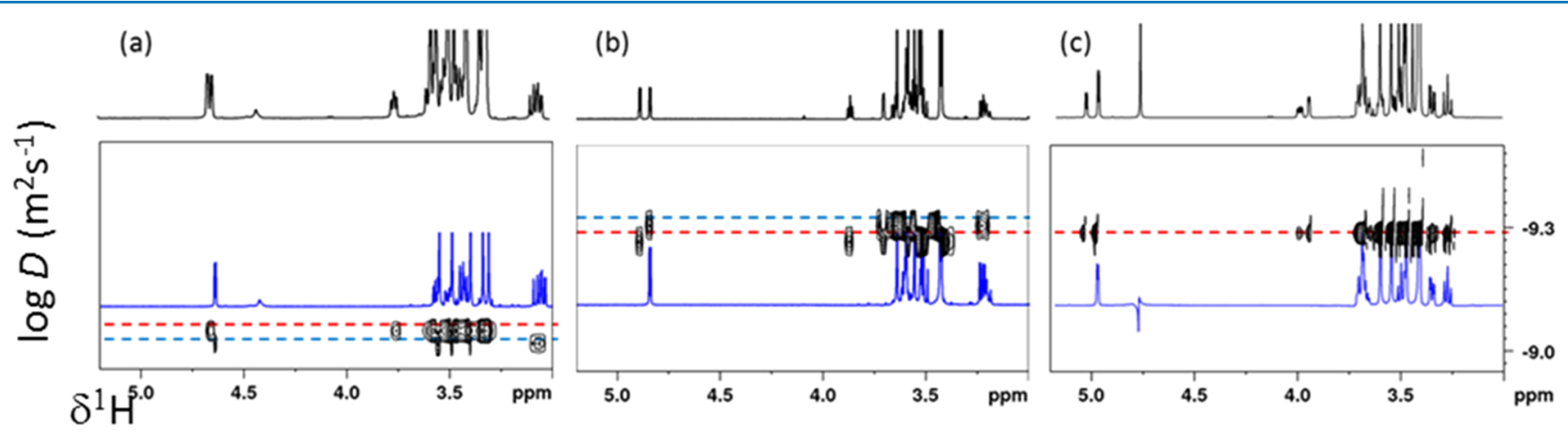

Figure 3. ${ }^{1} \mathrm{H}$-detected two-dimensional (2D) DOSY NMR spectra of $\alpha$-pMeGlc(1)/ $\alpha$-pMeGal(3) in (a) cyclohexane- $d_{12}$ (ca. $\left.40 \mathrm{mM}\right),(\mathrm{b}) \mathrm{CDCl}{ }_{3}$ (ca. $25 \mathrm{mM}$ ), and (c) $\mathrm{D}_{2} \mathrm{O}$ (ca. $25 \mathrm{mM}$ ). On top, the $1 \mathrm{D}{ }^{1} \mathrm{H}$ NMR spectra of the mixtures are displayed. The $1 \mathrm{D}{ }^{1} \mathrm{H}$ NMR spectrum of $\alpha$ pMeGlc(1) (blue) is also shown. Dotted lines identify the diffusion coefficient of each component (blue (1) and red (3)). 


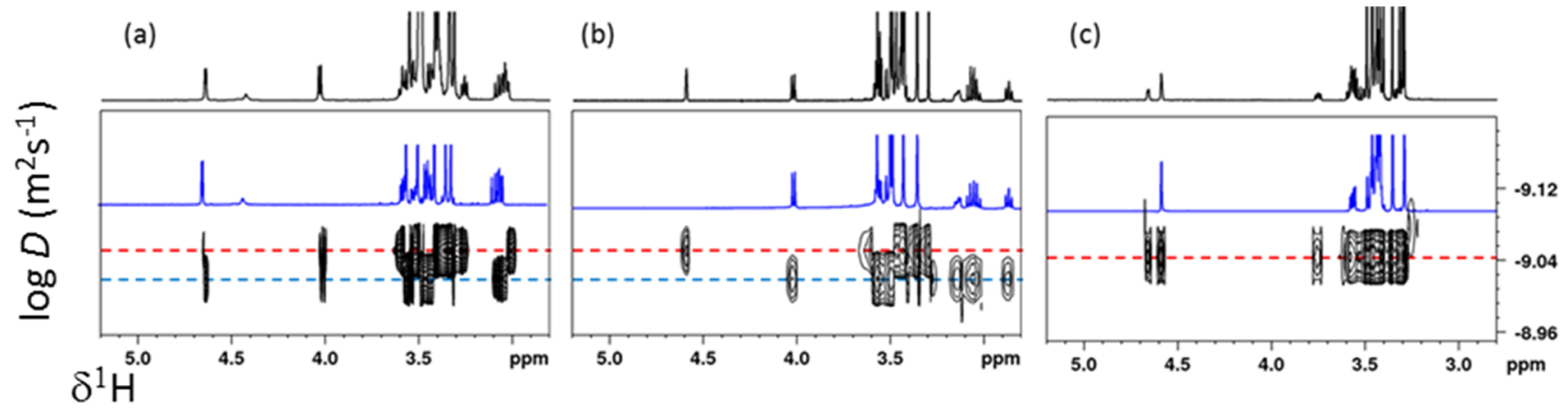

Figure 4. ${ }^{1} \mathrm{H}$-detected 2D DOSY NMR spectra of selected binary mixtures of permethylated monosaccharides $(\mathbf{1}-\mathbf{5})$ in cyclohexane- $d_{12}($ ca. 40 mM): (a) $\alpha$-pMeGlc(1)/ $\beta$-pMeGal(4), (b) $\beta$-pMeGlc(2) $/ \alpha$-pMeMan(5), (c) $\alpha$-pMeGal(3)/ $\alpha$-pMeMan(5). The $1 \mathrm{D}{ }^{1} \mathrm{H}$ NMR spectra of (a) $\alpha$ $\operatorname{pMeGlc}(\mathbf{1}),(\mathrm{b}) \beta$-pMeGlc(2), and (c) $\alpha$-pMeMan(5) are displayed in blue.

cyclohexane solutions, intermolecular hydrogen bonds in the aqueous mixtures, with water acting as either hydrogen bond donor or acceptor and compounds $\mathbf{1 - 5}$ as hydrogen bond acceptors, could make the hydrodynamic radius of the sugars rather similar, consequently yielding almost identical diffusion coefficients.

Thus, it appears that the separation of these sugar diastereomers by diffusion NMR depends on the properties of both, the solute and solvent. Hence, the apparent size of these sugars, as deduced by their corresponding coefficient diffusion values, is tuned by a complex balance between the sugar/solvent and solvent/solvent interactions.

Three's Company: Two Sugars and One Aromatic Cosolute. The previous results encouraged us to investigate whether the diffusion coefficient of the sugars, either in cyclohexane or in water, could also be altered by their specific interaction with an aromatic compound present in solution. The corroboration of this hypothesis could be taken as a novel although indirect proof of the formation of carbohydrate/ aromatic complexes via $\mathrm{CH}$-aromatic interactions.

First, the chemical shift perturbations (CSPs) of the NMR signals of the sugars upon addition of benzene to the mixtures in cyclohexane were detected. Interestingly, CSPs were noticeable for not only signals from those $\mathrm{CH}-\pi$ anticipated for the ring protons ${ }^{6}$ but also for methyl groups, suggesting the existence of $\mathrm{CH}_{3}-\pi$ interactions (Figure 5).

Next, the DOSY spectra of selected cyclohexane mixtures upon addition of benzene were acquired. Two representative results are presented in Figure 6. In general, the separation of most of the mixtures followed the same pattern as that in the absence of the aromatic molecules. The interval differences for the diffusion coefficients are, in general, only slightly larger, although noticeable in the presence of benzene, as exemplified in the DOSY spectrum recorded for the $\alpha$-pMeGlc $(1) / \alpha$ pMeGal(3)/benzene mixture in cyclohexane (Figure 6a). In contrast, the separation observed for the $\alpha$-pMeGal(3)/ $\alpha$ pMeMan(5) mixture in the presence of benzene was remarkable (Figure $6 \mathrm{~b}, D_{\mathrm{C}_{6} \mathrm{D}_{12}}(3)=9.29 \times 10^{-10} \mathrm{~m}^{2} \mathrm{~s}^{-1}$ and $D_{\mathrm{C}_{6} \mathrm{D}_{12}}(5)=10 \times 10^{-10} \mathrm{~m}^{2} \mathrm{~s}^{-1}$, respectively). Strikingly, these two sugars could not be separated in plain cyclohexane (Figure $4 c)$. Besides the observed $\mathrm{CH}_{3}-\pi$ interactions detected as CSP, the spatial arrangement of the $\mathrm{CH}$ groups of the $\alpha$-pMeGal(3) derivative fulfills the geometric requirements for establishing optimal multivalent $\mathrm{CH}-\pi$ interactions with aromatic rings (when compared with $\alpha$-pMeMan(5)). The putative stacking interactions of this molecule with benzene may satisfactorily

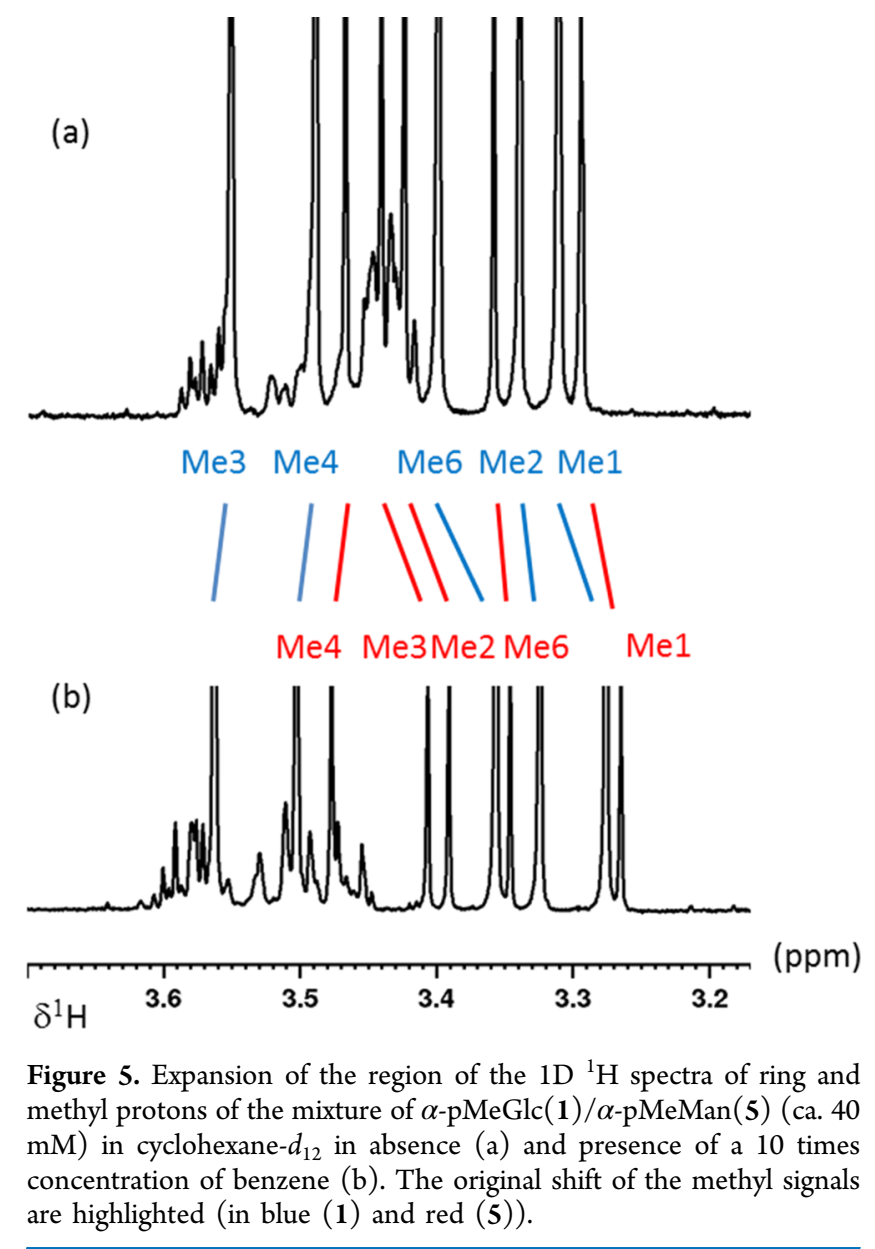

explain the apparent larger size of the $\alpha$-pMeGal(3) derivative in the presence of benzene when compared to that of the $\alpha$ $\mathrm{pMeMan}(5)$ analogue. Thus, for these systems in cyclohexane, the DOSY results provide an indirect proof of the existence of stabilizing $\mathrm{CH}-\pi$ and $\mathrm{Me}-\pi$ interactions between $\alpha$-pMeGal(3) and benzene.

Subsequently, the effect of the presence of an aromatic cosolute in aqueous mixtures was investigated. In contrast with the cyclohexane mixtures, major signal shifts were observed for the $\mathrm{CH}$-ring resonances when compared to those for the $\mathrm{O}$ methyl groups. The analysis of the DOSY spectra of the corresponding aqueous mixtures upon addition of phenol was, however, much less straightforward than the interpretation of the cyclohexane mixtures. In general, no clear separation was 


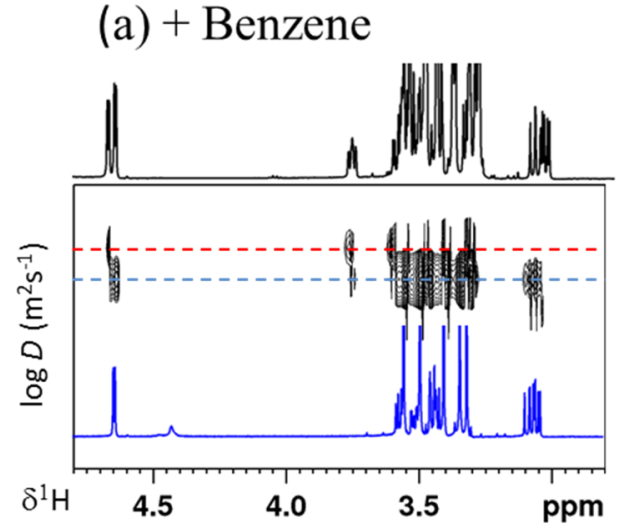

(b) + Benzene

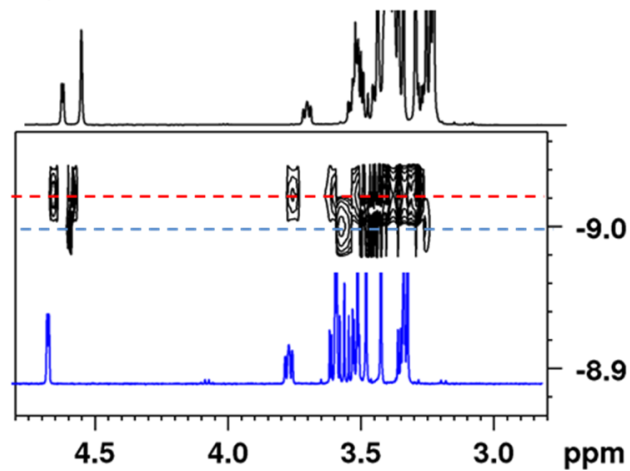

Figure 6. ${ }^{1} \mathrm{H} 2 \mathrm{D}$ DOSY spectra of the binary mixtures (a) $\alpha$-pMeGlc $(1) / \alpha$-pMeGal(3) and (b) $\alpha$-pMeGal(3)/ $\alpha$-pMeMan $(5)$ in cyclohexane- $d_{12}$ upon addition of benzene (ca. $40 \mathrm{mM})$. One-dimensional ${ }^{1} \mathrm{H}$ NMR spectra of one of the components of the mixture are displayed in blue [(a) $\alpha$ $\operatorname{pMeGlc}(\mathbf{1})$ and (b) $\alpha$-pMeGal(3)].
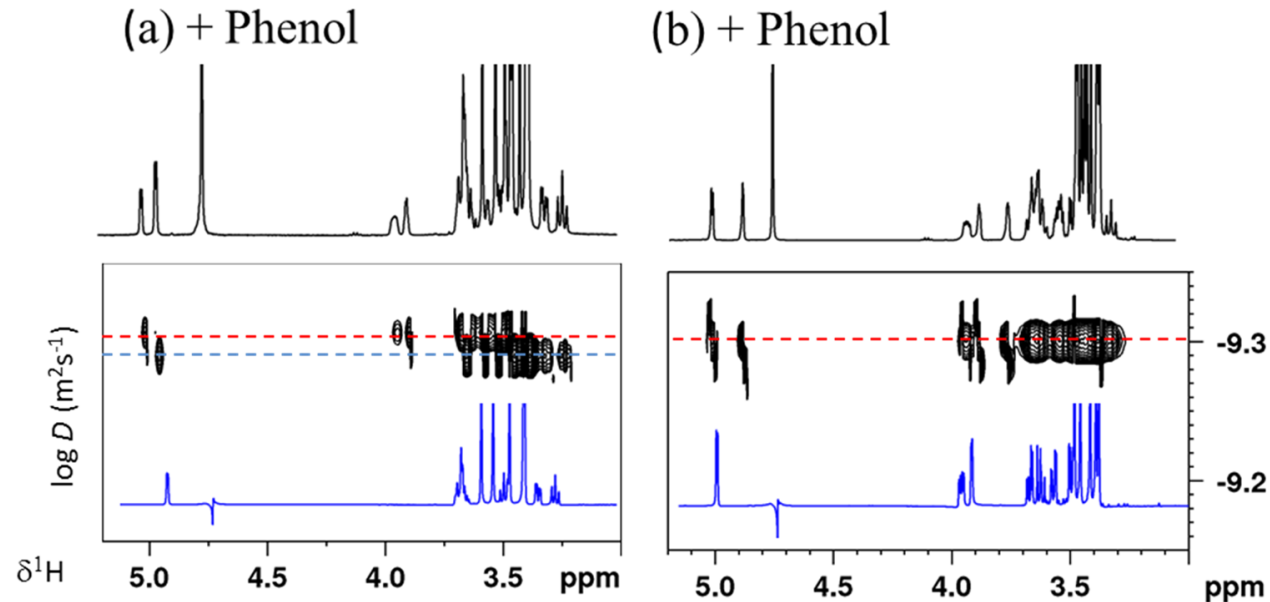

Figure 7. ${ }^{1} \mathrm{H} 2 \mathrm{D}$ DOSY spectra of the binary aqueous mixtures (a) $\alpha$-pMeGlc(1)/ $\alpha$-pMeGal(3) and (b) $\alpha$-pMeGal(3)/ $\alpha$-pMeMan $(5)($ ca. $25 \mathrm{mM})$ upon addition of phenol. One-dimensional ${ }^{1} \mathrm{H}$ spectra of one of the components of the mixture are displayed in blue [(a) (1) and (b) (3)].

achieved in the presence of phenol (Figure $7 \mathrm{~b}, D_{\mathrm{D}_{2} \mathrm{O}}(3)=$ $\left.D_{\mathrm{D}_{2} \mathrm{O}}(5)=4.91 \times 10^{-10} \mathrm{~m}^{2} \mathrm{~s}^{-1}\right)$ and only for the $\alpha$ $\operatorname{pMeGlc}(\mathbf{1}) / \alpha$-pMeGal(3)/phenol mixture, a well-defined separation of the diffusion coefficients of the sugars was observed (Figure $7 \mathrm{a}, D_{\mathrm{D}_{2} \mathrm{O}}(1)=5.02 \times 10^{-10} \mathrm{~m}^{2} \mathrm{~s}^{-1}$ and $\left.D_{\mathrm{D}_{2} \mathrm{O}}(3)=4.93 \times 10^{-10} \mathrm{~m}^{2} \mathrm{~s}^{-1}\right)$. In this case, the apparent hydrodynamic radius of $\alpha$-pMeGal(3) increased compared to that of $\alpha$-pMeGlc $(1)$ when phenol was present in solution. The smaller diffusion coefficient observed for $\alpha$-pMeGal(3) may be correlated with the tendency of $\alpha$-pMeGal(3) to display multivalent $\mathrm{CH}-\pi$ interactions through the $\mathrm{CH}$ groups at positions 3, 4, and 5. However, the possible interplay of intermolecular hydrogen bonds between both solutes and the solvent hamper the analysis of the observed data.

Therefore, although the data obtained for the cyclohexane mixtures allowed for a clear-cut discrimination of the components (separation by DOSY), the lack of separation for most of the investigated mixtures in water clearly suggests that the solvent properties strongly influence the type and strength of interactions between the species in solution. The intrinsic chemical nature of each sugar diastereomer in every specific mixture (solute/solvent interactions), as well as the balance between the intermolecular interactions (i.e., hydrogen bonds and $\mathrm{CH}-\pi$ interactions), modulates the recognition process.
Thus, for the $\mathrm{CH}-\pi$ contacts, solvophobic effects drive the interactions between the nonpolar groups. ${ }^{32}$

\section{CONCLUSIONS}

We have used diffusion NMR spectroscopy to screen the possibility of separation of the components of sugar binary mixtures in different solvents. The mixtures were composed by simple permethylated monosaccharide diastereomers. The working hypothesis relied on the existence of characteristic solvation/diffusion properties of each sugar that could be modulated by solute/solvent interactions and by the presence of aromatic rings (cosolute). Our results suggest that tiny configurational differences of the solutes (even at only one stereogenic center) drastically modify their interaction with the solvent, yielding different diffusion coefficients for the sugars in solution. Despite their obvious similarities (i.e., identical molecular weight and chemical functionalities), in nonpolar nonprotic cyclohexane, the glucose derivatives showed the smallest size and higher diffusion coefficient values compared to that of their counterparts in the mixture (galactose or mannose derivatives). Although self-diffusion coefficient values are very similar, noticeable differences were observed for the components of some of the studied mixtures. Intriguingly, the same mixtures in water (polar, protic solvent) could not be separated by DOSY, suggesting that their shapes and sizes (i.e., 
hydrodynamic radius of the sugars) become almost identical (similar diffusion coefficient) as a consequence of the interaction with the solvent (i.e., hydrogen bond). Further experiments with specific mixtures confirmed the influence of the solvent properties to properly achieve the challenging separation by diffusion NMR.

In addition, we have probed for the first time that sugararomatic interactions ( $\mathrm{CH}-\pi$ interactions) may modify the apparent size of monosaccharide derivatives and consequently their diffusion coefficients. This fact allows for the discrimination of the components of binary mixtures as a function of their respective ability to interact with aromatic compounds in solution. Moreover, we have shown the strong influence of the solvent properties to allow the detection of the $\mathrm{CH}-\pi$ interactions. The competition with other types of noncovalent interactions, such as hydrogen bonds, may definitively influence the attainable size and shape differences.

Overall, the presented results open up the possibility of using DOSY as an additional experimental tool to demonstrate and support the existence of intermolecular $\mathrm{CH}-\pi$ interactions during formation of specific complexes.

\section{METHODS}

NMR Samples. All permethylated monosaccharides were synthesized according to the literature description. ${ }^{26}$ All compounds used for the synthesis, as well as phenol, benzene, and deuterated solvents (i.e., $\mathrm{D}_{2} \mathrm{O}, \mathrm{CDCl}_{3}$, and cyclohexane$\left.d_{12}\right)$, were purchased from Sigma-Aldrich.

The $600 \mu \mathrm{L}$ NMR samples ( $5 \mathrm{~mm}$ NMR tubes) of the binary mixtures were prepared at a concentration of ca. $20-40 \mathrm{mM}$ for each sugar.

Stock solutions of aromatic compounds (i.e., phenol in $\mathrm{D}_{2} \mathrm{O}$ and benzene in cyclohexane- $d_{12}$ ) were prepared to add small volumes to the NMR samples to reach the desired sugar-aryl ratio concentrations.

The ${ }^{1} \mathrm{H}$ chemical shift scales are internally referred to trimethylsilylpropionate $\left(\mathrm{D}_{2} \mathrm{O}\right)$, tetramethylsilane $\left(\mathrm{CDCl}_{3}\right)$, and to the nondeuterated solvent signal ( $\delta 1.38 \mathrm{ppm})$ for cyclohexane- $d_{12}$ solutions. The ${ }^{13} \mathrm{C}$ chemical shift scales are referred to acetone $(\delta 30.9 \mathrm{ppm})$ in $\mathrm{D}_{2} \mathrm{O}$ and to the residual signal of chloroform $(\delta 77.4 \mathrm{ppm})$ and cyclohexane $(\delta 26.4$ ppm).

NMR Experiments. All experiments were performed at room temperature $(298 \mathrm{~K})$ either on a Bruker Avance (500 $\mathrm{MHz})$ or on a Bruker Avance II $(600 \mathrm{MHz})$ spectrometer, equipped with a triple resonance high-resolution probe (TXI). The characterization of the compounds in different solvents was achieved by NMR standard experiments. NMR experimental data and assignment of $\mathbf{1 - 5}$ in different solvents are available in the Supporting Information and at NMRshiftDB ${ }^{33}$ under the corresponding molecules identification codes (i.e., 60004073 (1), 60004029 (2), 60004074 (3), 60004071 (4), and $60004072(5))$. The attribution of scalar couplings was supported by ${ }^{1} \mathrm{H}$ NMR spectra simulation with SpinWorks 4.0.3 (K. Marat, University of Manitoba, 2014) using NUMRIT algorithms. $^{34}$

For the measurement of diffusion coefficients of the components of the mixtures, data acquisition and analysis were carried out using Bruker Topspin software (Bruker BioSpin, Germany). The DOSY experiments were performed using the longitudinal eddy current delay bipolar gradient pulse sequence (ledbpgp2s) from Bruker library. The diffusion delay $\Delta$ was set at $150-200 \mathrm{~ms}$, the gradient strength $g(2 \mathrm{~ms})$ was linearly incremented in 32-64 steps from 2 to $95 \%$ of its maximum value, longitudinal eddy currents delay of $5 \mathrm{~ms}$ and $8 / 16$ scans were recorded for each experiment. Processing was achieved using $4 \mathrm{~K}$ points in the $\mathrm{F} 2$ dimension and 256 points in F1. An exponential window function with $3 \mathrm{~Hz}$ line broadening was applied in the F2 dimension prior to Fourier transformation. After baseline correction, the diffusion dimension was processed with the DOSY processing program (Bruker TopSpin software 3.5). A logarithmic scaling was applied to the diffusion axis, and a noise sensitivity factor of 4 and line width factor of 1 were used. The fitting of the diffusion dimension in the 2D DOSY spectra was obtained using a single exponential fit.

\section{ASSOCIATED CONTENT}

\section{Supporting Information}

The Supporting Information is available free of charge on the ACS Publications website at DOI: 10.1021/acsomega.7b01630.

Assignment of the NMR $\left({ }^{1} \mathrm{H}\right.$ and $\left.{ }^{13} \mathrm{C}\right)$ spectra of compounds $\mathbf{1 - 5}$ in $\mathrm{D}_{2} \mathrm{O}$, cyclohexane- $d_{12}$, and $\mathrm{CDCl}_{3}$; Table S1 with values of diffusion coefficients (PDF)

\section{AUTHOR INFORMATION}

\section{Corresponding Authors}

*E-mail: jjbarbero@cicbiogune.es (J.J.-B.).

*E-mail: nils.schloerer@uni-koeln.de (N.E.S.).

*E-mail: mdiazher@uni-koeln.de (D.D.).

ORCID *

Gabriel Cuevas: 0000-0002-9528-133X

Jesús Jiménez-Barbero: 0000-0001-5421-8513

Notes

The authors declare no competing financial interest.

\section{ACKNOWLEDGMENTS}

The authors thank Kathrin König, Daniela Naumann, and Stefan Kuhn of the NMR facility of the University of Cologne for their technical support. D.D. and N.E.S. are very grateful to the University of Cologne as participants of the JobSharing project (for their support). L.J. was in part supported by the Deutsche Forschungsgemeinschaft (DFG) through the 1D NMR project (improvement of data quality in NMR, SCHL 580/3-1). The group at Spain thanks MINECO of Spain for financial support (Grants CTQ2015-64597-C2-1P and 2-P) and for the MINECO-Severo Ochoa Excellence Acreditation (SEV-2016-0644).

\section{REFERENCES}

(1) Gabius, H.-J.; Siebert, H.-C.; André, S.; Jiménez-Barbero, J.; Rüdiger, H. Chemical Biology of the Sugar Code. ChemBioChem 2004, $5,740-764$

(2) Spiwok, V. $\mathrm{CH} / \pi$ Interactions in Carbohydrate Recognition. Molecules 2017, 22, No. 1038.

(3) Hudson, K. L.; Bartlett, G. J.; Diehl, R. C.; Agirre, J.; Gallagher, T.; Kiessling, L. L.; Woolfson, D. N. Carbohydrate-Aromatic Interactions in Proteins. J. Am. Chem. Soc. 2015, 137, 15152-15160.

(4) Asensio, J. L.; Ardá, A.; Canada, F. J.; Jiménez-Barbero, J. Carbohydrate-Aromatic Interactions. Acc. Chem. Res. 2013, 46, 946954.

(5) Plevin, M. J.; Bryce, D. L.; Boisbouvier, J. Direct Detection of $\mathrm{CH} / \pi$ Interactions in Proteins. Nat. Chem. 2010, 2, 466-471.

(6) Fernández-Alonso, M. d. C.; Cañada, F. J.; Jiménez-Barbero, J.; Cuevas, G. Molecular Recognition of Saccharides by Proteins. Insights 
on the Origin of the Carbohydrate-Aromatic Interactions. J. Am. Chem. Soc. 2005, 127, 7379-7386.

(7) (a) Vandenbussche, S.; Díaz, D.; Fernández-Alonso, M. C.; Pan, W.; Vincent, S. P.; Cuevas, G.; Cañada, F. J.; Jiménez-Barbero, J.; Bartik, K. Aromatic-Carbohydrate Interactions: An NMR and Computational Study of Model Systems. Chem. - Eur. J. 2008, 14, 7570-7578. (b) Ramírez-Gualito, K.; Alonso-Ríos, R.; Quiroz-García, B.; Rojas-Aguilar, A.; Díaz, D.; Jiménez-Barbero, J.; Cuevas, G. Enthalpic Nature of the $\mathrm{CH} / \pi$ Interaction Involved in the Recognition of Carbohydrates by Aromatic Compounds, Confirmed by a Novel Interplay of NMR, Calorimetry, and Theoretical Calculations. J. Am. Chem. Soc. 2009, 131, 18129-18138.

(8) (a) Emenike, B. U.; Bey, S. N.; Bigelowa, B. C.; Chakravartulab, S. V. S. Quantitative Model for Rationalizing Solvent Effect in Noncovalent CH-Aryl Interactions. Chem. Sci. 2016, 7, 1401-1407. (b) Jiménez-Moreno, E.; Jimenez-Oses, G.; Gomez, A. M.; Santana, A. G.; Corzana, F.; Bastida, A.; Jimenez-Barbero, J.; Asensio, J. L. A Thorough Experimental Study of $\mathrm{CH} / \pi$ Interactions in Water: Quantitative Structure-Stability Relationships for Carbohydrate/ Aromatic Complexes. Chem. Sci. 2015, 6, 6076-6085.

(9) Bautista-Ibáñez, L.; Ramírez-Gualito, K.; Quiroz-García, B.; Rojas-Aguilar, A.; Cuevas, G. Calorimetric Measurement of the $\mathrm{CH} / \pi$ Interaction Involved in the Molecular Recognition of Saccharides by Aromatic Compounds. J. Org. Chem. 2008, 73, 849-857.

(10) (a) Novoa-Carballal, R.; Fernandez-Megia, E.; Jimenez, C.; Riguera, R. NMR methods for Unravelling the Spectra of Complex Mixtures. Nat. Prod. Rep. 2011, 28, 78-98. (b) Cohen, Y.; Avram, L.; Frish, L. Diffusion NMR Spectroscopy in Supramolecular and Combinatorial Chemistry: An Old Parameter-New Insights. Angew. Chem., Int. Ed. 2005, 44, 520-554.

(11) Jones, J. A.; Wilkins, D. K.; Smith, L. J.; Dobson, C. M. Characterization of Protein Unfolding by NMR Diffusion Measurements. J. Biomol. NMR 1997, 10, 199-203.

(12) Ambrus, A.; Yang, D. Diffusion-Ordered NMR Spectroscopy for Analysis of DNA Secondary Structural Elements. Anal. Biochem. 2007, $367,56-67$.

(13) Li, D.; Keresztes, I.; Hopspon, R.; Williard, P. G. Characterization of Reactive Intermediates by Multinuclear Diffusion-Ordered NMR Spectroscopy (DOSY). Acc. Chem. Res. 2009, 42, 270-280.

(14) (a) Viel, S.; Mannina, L.; Segre, A. Detection of a $\pi-\pi$ Complex by Diffusion-Ordered Spectroscopy (DOSY). Tetrahedron Lett. 2002, 43, 2515-2519. (b) Yao, S.; Howlett, G. J.; Norton, R. S. Peptide SelfAssociation in Aqueous Trifluoroethanol Monitored by Pulsed Field Gradient NMR Diffusion Measurements. J. Biomol. NMR 2000, 16, 109-119.

(15) Kapur, G. S.; Cabrita, E. J.; Berger, S. The Qualitative Probing of Hydrogen Bond Strength by Diffusion-Ordered NMR Spectroscopy. Tetrahedron Lett. 2000, 41, 7181-7185.

(16) Cabrita, E. J.; Berger, S.; Brauer, P.; Kärger, J. High-Resolution DOSY NMR with Spins in Different Chemical Surroundings: Influence of Particle Exchange. J. Magn. Reson. 2002, 157, 124-131.

(17) Díaz, M. D.; Berger, S. Studies of the Complexation of Sugars by Diffusion-Ordered NMR Spectroscopy. Carbohydr. Res. 2000, 329, 15.

(18) (a) Balayssac, S.; Delsuc, M. A.; Gilard, V.; Prigent, Y.; MaletMartino, M. Two-Dimensional DOSY Experiment with Excitation Sculpting Water Suppression for the Analysis of Natural and Biological Media. J. Magn. Reson. 2009, 196, 78-83. (b) Guerrero-Martınez, A.; Palafox, M. A.; Tardajos, G. Unexpected Binding Mode of Gemini Surfactants and $\gamma$-Cyclodextrin: DOSY as a Tool for the Study of Complexation. Chem. Phys. Lett. 2006, 432, 486-490.

(19) Tomati, U.; Belardinelli, M.; Galli, E.; Iori, V.; Capitani, D.; Mannina, L.; Viel, S.; Segre, A. NMR Characterization of the Polysaccharidic Fraction from Lentinula Edodes Grown on Olive Mill Waste Waters. Carbohydr. Res. 2004, 339, 1129-1134.

(20) (a) Balayssac, S.; Trefia, S.; Gilarda, V.; Malet-Martino, M.; Martino, R.; Delsuc, M. A. 2D and 3D DOSY 1H NMR, a Useful Tool for Analysis of Complex Mixtures: Application to Herbal Drugs or Dietary Supplements for Erectile Dysfunction. J. Pharm. Biomed. Anal.
2009, 50, 602-612. (b) Malet-Martino, M.; Holzgrabe, U. NMR Techniques in Biomedical and Pharmaceutical Analysis. J. Pharm. Biomed. Anal. 2011, 55, 1-5.

(21) Macchioni, A.; Ciancaleoni, G.; Zuccaccia, C.; Zuccaccia, D. Determining Accurate Molecular Sizes in Solution Through NMR Diffusion Spectroscopy. Chem. Soc. Rev. 2008, 37, 479-489.

(22) (a) Groves, P.; Palczewska, M.; Molero, M. D.; Batta, G.; Cañada, F. J.; Jiménez-Barbero, J. Protein Molecular Weight Standards can Compensate Systematic Errors in Diffusion-Ordered Spectroscopy. Anal. Biochem. 2004, 331, 395-397. (b) Viel, S.; Capitani, D.; Mannina, L.; Segre, A. Diffusion-Ordered NMR Spectroscopy: A Versatile Tool for the Molecular Weight Determination of Uncharged Polysaccharides. Biomacromolecules 2003, 4, 1843-1847.

(23) (a) Reile, I.; Aspers, R. L. E. G.; Feiters, M. C.; Rutjesa, F. P. J. T.; Tessari, M. Resolving DOSY Spectra of Isomers by Methanol-d4 Solvent Effects. Magn. Reson. Chem. 2017, 55, 759-762. (b) Codling, D. J.; Zheng, G.; Stait-Gardner, T.; Yang, S.; Nilsson, M.; Price, W. S. Diffusion Studies of Dihydroxybenzene Isomers in Water-Alcohol Systems. J. Phys. Chem. B 2013, 117, 2734-2741.

(24) Adams, R. W.; Aguilar, J. A.; Cassani, J.; Morris, G. A.; Nilsson, M. Resolving Natural Product Epimer Spectra by Matrix-Assisted DOSY. Org. Biomol. Chem. 2011, 9, 7062-7064.

(25) (a) Pages, G.; Delaurent, C.; Caldarelli, S. Simplified Analysis of Mixtures of Small Molecules by Chromatographic NMR Spectroscopy. Angew. Chem., Int. Ed. 2006, 45, 5950-5953. (b) Viel, S.; Ziarelli, F.; Caldarelli, S. Enhanced Diffusion-Edited NMR Spectroscopy of Mixtures using Chromatographic Stationary Phases. Proc. Natl. Acad. Sci. U.S.A. 2003, 100, 9696-9698. (c) Carrara, C.; Viel, S.; Delaurent, C.; Ziarelli, F.; Excoffier, G.; Caldarelli, S. Chromatographic NMR in NMR Solvents. J. Magn. Reson. 2008, 194, 303-306. (d) Hoffman, R. E.; Arzuan, H.; Pemberton, C.; Aserin, A.; Garti, N. High-Resolution NMR "Chromatography" using a Liquids Spectrometer. J. Magn. Reson. 2008, 194, 295-299. (e) Joyce, R. E.; Day, I. J. Chromatographic NMR with Size Exclusion Chromatography Stationary Phases. J. Magn. Reson. 2012, 220, 1-7.

(26) (a) Wang, H.; Sun, L.; Glazebnik, S.; Zhao, K. Peralkylation of Saccharides under Aqueous Conditions. Tetrahedron Lett. 1995, 36, 2953-2956. (b) Käsbeck, L.; Kessler, H. Convenient Syntheses of 2,3,4,6-Tetra-O-Alkylated D-Glucose and D-Galactose. Liebigs Ann. 1997, 169-173.

(27) Kählig, H.; Dietrich, K.; Dömer, S. Analysis of Carbohydrate Mixtures by Diffusion Difference Spectroscopy. Monatsh. Chem. 2002, 133, 589-598.

(28) Monteiro, C.; Herve du Penhoat, C. Translational Diffusion of Dilute Aqueous Solutions of Sugars as Probed by NMR and Hydrodynamic Theory. J. Phys. Chem. A 2001, 105, 9827-9833.

(29) (a) Ekdawi-Sever, N.; de Pablo, J. J.; Feick, E.; von Meerwal, E. Diffusion of Sucrose and $\alpha, \alpha$-trehalose in Aqueous Solutions. J. Phys. Chem. A 2003, 107, 936-943. (b) Colbourne, A. A.; Meier, S.; Morris, G. A.; Nilsson, M. Unmixing the NMR Spectra of Similar Species vive la Difference. Chem. Commun. 2013, 49, 10510-10512.

(30) (a) de Vries, N. K.; Buck, H. M. Different Rotamer Populations Around the C-5-C-6 Bond for $\alpha$ - and $\beta$-d-Galactopyranosides through the Combined Interaction of the Gauche and Anomeric Effects: A 300-MHz 1H-n.m.r. and mndo Study. Carbohydr. Res. 1987, 165, 116. (b) Vogt, D. C.; Stephen, A. M.; Jackson, G. E. 1H-N.m.r. and 13C-n.m.r. Spectroscopy of Methyl Ethers of D-Galactopyranose. Carbohydr. Res. 1990, 206, 333-339. (c) Haverkamp, J.; van Dangen, J. P. C. M.; Vliegenhart, J. F. G. PMR and CMR Spectroscopy of Methyl 2,3,4,6-Tetra-O-Methyl- $\alpha$ and $\beta$-D-Glucopyranoside: An Application to the Identification of Partially Methylated Glucoses. Tetrahedron 1973, 29, 3431-3439. (d) Rockwell, G. D.; Grindley, T. Effect of Solvation on the Rotation of Hydroxymethyl Groups in Carbohydrates. J. Am. Chem. Soc. 1998, 120, 10953-10963.

(31) Nóbrega, C.; Vazquez, J. T. Conformational Study of the Hydroxymethyl Group in $\alpha$-D-Mannose Derivatives. Tetrahedron: Asymmetry 2003, 14, 2793-2801.

(32) (a) Adam, C.; Yang, L.; Cockroft, S. L. Quantitative Model for Rationalizing Solvent Effect in Noncovalent $\mathrm{CH}$-Aryl Interactions. 
Angew. Chem., Int. Ed. 2015, 54, 1164-1167. (b) Yang, L.; Adam, C.; Cockro, S. L. Quantifying Solvophobic Effects in Nonpolar Cohesive Interactions. J. Am. Chem. Soc. 2015, 137, 10084-10087.

(33) Kuhn, S.; Schlörer, N. E. Facilitating Quality Control for Spectra Assignments of Small Organic Molecules: nmrshiftdb2-a Free InHouse NMR Database with Integrated LIMS for Academic Service Laboratories. Magn. Reson. Chem. 2015, 53, 582-589.

(34) Martin, J. S.; Quirt, A. R. NMR Spectra of Symmetric Molecules. I. The spin Hamiltonian for Twofold Symmetry. J. Magn. Reson. 1971, 5, 318-327 (modified by Marat K. and Sebastian R, SpinWorks 4, University of Manitoba). 